\title{
Neonatal Factors Associated with HIV Long Term Non-Progressors in a Cohort of Vertically Infected Children in Rio de Janeiro, Brazil ("Peixe" Project)
}

\author{
Hofer, Cristina B.; Oliveira, Ricardo Hugo; Machado, Elizabeth S.; Pala, Alessandra; Evangelista, Lúcia; Fernandes, Iraina; and \\ Abreu, Thalita F. \\ Universidade Federal do Rio de Janeiro; Rio de Janeiro, RJ, Brazil
}

\begin{abstract}
There are only scarce data on HIV progression in vertically infected children in developing countries. The aim of this study is to describe factors from neonatal period associated with long term non-progression (LTNP), in a Brazilian cohort. A cohort study, with data systematically collected from the "Peixe" Cohort (cohort study of children conducted at the main HIV Pediatric Center in Rio de Janeiro, from 1996 to 2005). The study included children who were vertically infected and started follow up at 5 years of age or younger. LTNP, defined as not reaching category $\mathrm{C}$ or severe immunosuppression before 5 years of age. Neonatal and demographic factors were studied. Variables with $\mathbf{p}$-value $<\mathbf{0 . 1 5}$ were included in a logistic regression model. 213 patients were included, of whom $42 \%(89 / 213)$ were classified as LTNP. Variables independently associated with LTNP were: baseline (at study entry) CD4+ cells (per \%) $(\mathrm{OR}=1.06,95 \% \mathrm{CI}=1.01-1.12)$; age of initiating follow-up, per month $(\mathrm{OR}=1.03,95 \% \mathrm{CI}=1.01$ 1.06); $\mathrm{ZDV}$ use duriing newborn period $(\mathrm{OR}=3.31,95 \% \mathrm{CI}=0.86-12.71)$; use of antiretroviral (ART) before classification $\mathrm{C}$ or severe immunosuppression $(\mathrm{OR}=5.89,95 \% \mathrm{CI}=2.03-17.10)$. Adjusting for age at the beginning of follow-up, antiretroviral that was unsuccessfully used to prevent maternal-to-child transmission (ZDV use in neonatal period) was associated with better prognosis.
\end{abstract}

ARTs initiation before category $C$ or severe immunosuppression was also associated with LTNP.

Key-Words: HIV, antiretroviral therapy, long term non-progressors, children, Brazil.

It is estimated that $20-40 \%$ of HIV-1-infected children present with rapid disease progression and develop AIDS within the first years of life [1-4]. On the other hand, $60-80 \%$ of infected children manifest less severe symptoms and many survive through adolescence [3-6]. In developed countries, advanced maternal disease, high maternal viral load, and maternal and infant immune suppression were associated with rapid HIV progression in children [7]. In developing countries, where the majority of perinatal infections occur, there are only scarce data on HIV progression and risk factors. Meanwhile, in some developed countries, all HIV infected children are treated with highly active antiretroviral therapy (HAART) in early infancy. [8]. Brazilian guidelines [9] recommend that HIV infected infants should be treated only if they present any stage $\mathrm{B}$ or $\mathrm{C}$ symptoms and/or are in immune categories 2 or 3 (CDC) [10]. Although there is some evidence that infants benefit from treatment started in their first year of life, with early HIV replication control and preservation of the immune system $[3,4,11]$, aspects associated with HAART adverse events, adherence and emergence to drug resistant virus must also be considered [5]. Another important issue is the possibility that almost 50\% [1-4] of these infants would be long-term nonprogressors (LTNP), and would not benefit from HAART until five years of age or older. It is important

Received on 15 March 2009; revised 6 July 2009.

Address for correspondence: Dr. Cristina Barroso Hofer. Rua Lopes Quintas, 340/106 Jardim Botanico RJ Brazil - Zip code:22460-010. Email: cbhofer@hucff.ufrj.br, cbhst13br@yahoo.com. This project was supported by Conselho Nacional de Pesquisa (CNPq)-grant 403373/ 04-2. This study was reviewed and approved by the Instituto de Puericultura e Pediatria Martagão Gesteira Ethical Committee.

The Brazilian Journal of Infectious Diseases 2009;13(4):276-279. (C) 2009 by The Brazilian Journal of Infectious Diseases and Contexto Publishing. All rights reserved. to identify early life events that might be associated with long-term nonprogressors, so HAART could be individualized even among infants. The aim of this study is to evaluate neonatal characteristics associated with LTNP in a Brazilian cohort.

\section{Material and Methods}

Data were systematically collected from the "Peixe" Project - a cohort study of children conducted at an HIV Pediatric Center in Rio de Janeiro, Brazil, Instituto de Puericultura e Pediatria Martagão Gesteira (IPPMG), from 1996 through 2005. Rio de Janeiro is the second largest city in Brazil and since 1995573 cases of AIDS in children were notified in this city. Since 1996, all children who were followed up at the IPPMG were enrolled in a prospective cohort study. During their appointments, the health care worker (HCW) who assisted the child was responsible for filling out the cohort forms. The HCW used a pre-validated standardized questionnaire, with demographic, social, epidemiological, clinical, laboratory, and medication data. Prenatal and perinatal data from children who were not followed up in this center since their birth were extracted from maternal prenatal card provided in any health care center in Brazil, where all prenatal and perinatal information are registered and is a very reliable information source. Visits were scheduled at least three times a year, with laboratory evaluations at least twice a year. Children were followed up at least until the age of 13, when they are referred to an adolescent clinic. Children who were vertically infected and started follow up at 5 years of age or younger were included in this study. Exclusion criteria were: lack of birth information or follow-up as an outpatient for less than 1 year.

The main endpoint was LTNP, defined as not reaching category $\mathrm{C}$ and/or severe immunosuppression (stage 3) [10] before the age of five years. Early-progressors (EP) were 
defined as reaching category $C$ and/or severe immunosuppression (stage 3) [10] before the age of five years Neonatal and demographic factors were studied and compared between LTNP and EP.

\section{Statistical Analysis}

We evaluated possible risk factors associated with LTNP. Data analysis was performed using Stata version 8.0 statistical software (Stata Corp., College Station, TX). Bivariate analysis was performed using independent t-test (for variables with a normal distribution) or Wilcoxon (Mann-Whiteney) twosample test (for variables that did not follow the normal distribution). The Chi-square test was used to evaluate associations for categorical variables (or Fisher Exact Test, if $20 \%$ or higher of the table cells have an expected value 5 or lower). Variables with a p-value $<0.15$ were included in the multivariate analysis.

A main-effects logistic regression model was fitted using the stepwise maximum likelihood estimation technique. The level of significance for removal of a variable in backward regression was 0.10 , and forward regression was 0.05 . Interactions were assessed using the -2 log likelihood ratio test to compare models with and without interactions. The Pearson's Chi-square goodness of fit test, as well as the Hosmer-Lemeshow test, were used to evaluate fitness of the model.

\section{Results}

Among 213 patients included, 27\% (57/213) were born before 1994. 51\% (108/213) were males, 54\% (115/213) were considered as non-white (i.e., primarily of African descent), mean birth weight was 3108g, mean age at diagnosis was 21.7 months (ranging 0-59 months), and mean age at which they started follow-up at our Center was 24.7 months (ranging 0-59 months). 42\% (89/213) were classified as LTNP. Several factors were associated with LTNP, such as gender, use of intravenous Zidovudine (ZDV) during labor, ZDV use during the newborn period, no history of breastfeeding, age at follow-up initiation, baseline (at study entry) CD4+ cells count and viral load, use of antiretrovirals (ART) before class $\mathrm{C}$ diagnosis condition or severe immunosuppression, and use of ART during pregnancy (Table 1).

In the multivariate analysis, factors positively associated with LTNP were: older age at follow-up initiation, higher baseline CD4+ cells (first CD4+ cells count available), ZDV use during the newborn period, and ART use before classification $\mathrm{C}$ or severe immunosuppression (Table 2).

\section{Discussion}

Factors associated with HIV pathogenesis (higher baseline CD4+ cells count) as well as factors associated with care (ZDV use during the neonatal period, start of ART before patient reaches class $\mathrm{C}$ diagnosis condition or severe immunosuppression) were related to LTNP in the 9-year cohort study.
In this cohort, the introduction of ART before patients reached CDC categories $\mathrm{C}$ and/or 3 was associated with LTNP. Soh et al. also demonstrated that initiation of proteaseinhibitor-based combination therapy before severe immunosuppression was associated with better CD4+ cells recovery [12]. In our study, we also demonstrated that even in patients with higher CD4+ cells, the clinical evidence of immunosuppression (category C) is important to consider when evaluating the exact point to start on HAART.

The use of ZDV during the neonatal period was associated with LTNP, even when adjusting for age at follow-up initiation. The use of ART during pregnancy, labor, and the neonatal period was associated with an important decrease in maternalto-child transmission (MTCT) [13]. These interventions may result in transmission of a resistant HIV strain to the newborn, who then fails prevention strategies, and may also jeopardize the child's future treatment options [14-16]. In this study we demonstrated that the use of ZDV during the neonatal period was associated with a good long term prognosis. In another study [7], it was associated with a poorer short term prognosis in the bivariate analysis, but when data were adjusted for variables associated with maternal viral load and CD4+ cells count, it was not statistically significant. One hypothesis for the better prognosis found in our study is that exposure to ZDV in the neonatal period may induce viral mutations, leading to a fitness-reduced viral strain [17-19].

Two other studies evaluated the impact of the PACTG076 protocol in children's prognosis at 18 months [20] or 3 years [21], with divergent results. In the first, a retrospective study, a worse prognosis was found for children who were exposed to ZDV perinatally. In the second, a prospective study, a better prognosis was found for those who started perinatal ZDV. However, this study also found a better prognosis for patients who started earlier ART, and the authors argued that perhaps infants exposed to ZDV perinatally had more contact with the health care system, started earlier ART, and thus had a better prognosis. In this study, probably due to sample size issues, the authors were not able to adjust for it. In our data, we were able to adjust for this possible bias, and we confirmed the findings that neonatal exposure to ZDV is associated with a better long-term prognosis.

The main limitations of our data are that we were not able to retrieve all neonatal information, since some children were adopted or the neonatal files were incomplete or lost, and 27\% were born before 1994, before the Protocol PACTG076. On the other hand, this cohort study was derived from a population followed up for a long period, in a systematic matter, in a middle-income developing country where children have access to ARTs. We were able to study separately the three parts of the PACTG076 Protocol; use of ZDV at prenatal, perinatal, and neonatal periods, and newborn use of ZDV was important, even when adjusting for age at ART initiation.

Another important limitation is the fact that not all the children were followed since birth, and the results could be biased by children who died before reach follow up or children 
Table 1. Bivariate analysis - Factors Associated with LTNP.

\begin{tabular}{lccr}
\hline Variable & LTNP-Total (\%) & EP-Total (\%) & p-value \\
\hline Gender - male & $36 / 89(40)$ & $69 / 129(53)$ & 0.03 \\
Race - white & $42 / 77(54)$ & $46 / 113(41)$ & 0.06 \\
HIV infected father & $41 / 65(63)$ & $47 / 75(63)$ & 0.96 \\
Mother IV drug user & $10 / 82(12)$ & $9 / 114(8)$ & 0.34 \\
Maternal age at birth $\leq 21$ & $19 / 82(23)$ & $32 / 114(28)$ & 0.44 \\
Vaginal birth & $53 / 83(64)$ & $76 / 120(63)$ & 0.94 \\
Not breastfed & $20 / 85(23)$ & $11 / 122(9)$ & $<0.01$ \\
Prenatal care & $68 / 82(83)$ & $104 / 121(86)$ & 0.56 \\
ART use during pregnancy & $9 / 88(10)$ & $4 / 120(3)$ & 0.07 \\
ZDV IV during labor & $8 / 88(9)$ & $4 / 120(3)$ & 0.07 \\
Newborn used ZDV & $19 / 88(22)$ & $10 / 124(8)$ & $<0.01$ \\
Weight at birth - mean (grams) & 3149 & 3064 & 0.33 \\
Age of initiating follow up - mean (months) & 26.32 & 20.81 & 0.04 \\
Baseline CD4+ cells - mean (\%) & 23.09 & 15.89 & $<0.01$ \\
Baseline viral load - mean (copies/mL) & 367,854 & 889,357 & 0.05 \\
ART before classification C or severe immunosupression & $40 / 89(45)$ & $13 / 111(12)$ & $<0.01$ \\
\hline
\end{tabular}

Table 2. Multivariate analysis - Factors Associated with LTNP.

\begin{tabular}{lccc}
\hline Variable & OR & p-value & 95\%Confidence interval \\
\hline Age of initiating follow up (per month) & 1.03 & 0.03 & $1.01-1.06$ \\
Newborn used ZDV & 3.31 & 0.08 & $0.86-12.71$ \\
Baseline CD4+ cells (per \%) & 1.06 & 0.03 & $1.01-1.12$ \\
ART before classification C or severe immunosupression & 5.89 & $<0.01$ & $2.03-17.10$ \\
\hline
\end{tabular}

who were not diagnosed. However, this study sample size (more than half of cases notified in Rio de Janeiro after 1995) probably is representative from this city HIV infected children. And even if this bias is inserted, this study would demonstrate factors associated with non-progressors in a group of patients followed up in our clinic, and who would probably be the same population frame from other clinics.

In conclusion, we were able to demonstrate that some factors associated with the perinatal/newborn period, such as baseline CD4+ cells count and ZDV use, were associated with a better prognosis, even adjusting for ART use before reaching $C$ and/or 3 category, or the length of follow-up.

\section{Acknowledgements}

Conselho Nacional de Pesquisa do Brasil (CNPq)-grant 403373/04-2, for supporting this research. The CNPq had no role in the study design, in the collection, analysis and interpretation of data; in the writing of the manuscript; and in the decision to submit the manuscript for publication.

\section{References}

1. European Collaborative Study. Fluctuations in symptoms in human immunodeficiency virus-infected children: the first 10 years of life. Pediatrics 2001;108:116-22.

2. Scott GB, Hutto C, Makuch RW, Mastrucci MT, O’Connor T, Mitchell CD, et al. Survival in children with perinatally acquired human immunodeficiency virus type 1 infection. $N$ Engl J Med
1989; 321:1791-6.

3. Foster C. \& Lyall EG. Children with HIV: improved mortality and morbidity with combination antiretroviral therapy. Current Opinion in Infectious Diseases 2005; 18:253-9.

4. Walker AS. Doerholt K. Sharland M. Gibb DM. Collaborative HIV Paediatric Study (CHIPS) Steering Committee. Response to highly active antiretroviral therapy varies with age: the UK and Ireland Collaborative HIV Paediatric Study. AIDS 2004; 18:1915-24.

5. Aboulker JP. Babiker A. Chaix ML. Compagnucci A. Darbyshire J. Debre M. Faye A. Giaquinto C. Gibb DM. Harper L. Saidi Y. Walker AS. Paediatric European Network for Treatment of AIDS. Highly active antiretroviral therapy started in infants under 3 months of age: 72-week follow-up for CD4 cell count, viral load and drug resistance outcome. AIDS 2004; 18:237-45.

6. Abrams EJ, Thomas P, Weedon J, Bertolli J, Bornschegle K, Cervia $\mathrm{J}$, et al. Aging cohort of perinatally human immunodeficiency virus-infected children in New York City. Pediatr Infect Dis J 2001; 20:511-7.

7. Rich KC. Fowler MG. Mofenson LM. Abboud R. Pitt J. Diaz C. Hanson IC. Cooper E. Mendez H. Maternal and infant factors predicting disease progression in human immunodeficiency virus type 1-infected infants. Women and Infants Transmission Study Group. Pediatrics 2000; 105:e8.

8. Guidelines for the Use of Antiretroviral Agents in Pediatric HIV Infection - Working Group on Antiretroviral Therapy and Medical Management of HIV-Infected Children, 2005. In: http:/ /aidsinfo.nih.gov/ContentFiles/PediatricGuidelines.pdf.

9. Guia de Tratamento Clínico da Iinfecção pelo HIV em Pediatria Ministério da Saúde do Brasil, 2006.In:http://www.aids.gov.br/ $\mathrm{d}$ a t a / d o c u m e n t s/stored D o c u m e n t s / consenso_crianca_versao_preliminar_25ago06.pdf. 
10. Centers for Disease Control and Prevention. Revised classification system for human immunodeficiency virus infection in children less than 13 years of age. MMWR, 1994; 43: (No. RR-12):. 110 .

11. Violari A.1, Cotton M.2, Gibb D.3, Babiker A.3, Steyn J.1, JeanPhillip P.4, McIntyre J.1, on behalf of the CHER Study Team. Antiretroviral therapy initiated before 12 weeks of age reduces early mortality in young HIV-infected infants: evidence from the Children with HIV Early Antiretroviral Therapy (CHER) Study. 4th IAS Conference on HIV Pathogenesis, Treatment and Prevention, Abstract no. WESS103, Sidney 2007.

12. Soh CH. Oleske JM. Brady MT. Spector SA. Borkowsky W. Burchett SK. Foca MD. Handelsman E. Jimenez E. Dankner WM. Hughes MD. Pediatric AIDS Clinical Trials Group. Longterm effects of protease-inhibitor-based combination therapy on CD4 T-cell recovery in HIV-1-infected children and adolescents. Lancet 2003; 362: 2045-51.

13. Connor EM et al. Reduction of maternal-infant transmission of human immunodeficiency virus type 1 with zidovudine treatment. New England Journal of Medicine 1994; 331: 117380 .

14. Parker MM. Wade N. Lloyd RM Jr. Birkhead GS. Gallagher BK. Cheku B. Sullivan T. Taylor J. Prevalence of genotypic drug resistance among a cohort of HIV-infected newborns. Journal of Acquired Immune Deficiency Syndromes: JAIDS 2003; 32: 292-7.

15. Desai N. Mathur M. Selective transmission of multidrug resistant HIV to a newborn related to poor maternal adherence. Sexually Transmitted Infections 2003; 79: 419-21, 2003.
16. Fowler MG. Mofenson L. McConnell M. The interface of perinatal HIV prevention, antiretroviral drug resistance, and antiretroviral treatment: what do we really know? Journal of Acquired Immune Deficiency Syndromes: JAIDS. 2003; 34:308-11, 2003.

17. Prado JG. Franco S. Matamoros T. Ruiz L. Clotet B. MenendezArias L. Martinez MA. Martinez-Picado J. Relative replication fitness of multi-nucleoside analogue-resistant HIV-1 strains bearing a dipeptide insertion in the fingers subdomain of the reverse transcriptase and mutations at codons 67 and 215. Virology 2004; 326:103-12.

18. Isaguliants MG. Belikov SV. Starodubova ES. Gizatullin RZ. Rollman E. Zuber B. Zuber AK. Grishchenko OI. Rytting AS. Kallander CF. Kochetkov SN. Karpov VL. Wahren B. Mutations conferring drug resistance affect eukaryotic expression of HIV type 1 reverse transcriptase. AIDS Research \& Human Retroviruses 2004; 20:191-201.

19. Bennett M E j D, Heneine W, and Garcia-Lerma JG. Mutation Interactions Modulate the Fitness Cost of Drug Resistance Mutations in Transmitted Isolates: Implications for Persistence of Transmitted Resistance - Poster no. 627, In: 13th Conference on Retrovirus and Opportunistic Infections, Denver, 2006.

20. de Souza RS, Gomez-Marin O, Scott GB, Guasti S, O’Sullivan MJ, Oliveira RH, Mitchell CD. Effect of prenatal zidovudine on disease progression in perinatally HIV-1-infected infants. J Acquir Immune Defic Syndr 2000; 24:154-61.

21. Berk DR, Falkovitz-Halpern MS, Sullivan B, Ruiz J, Maldonado YA. Disease Progression among HIV-Infected Children who Received Perinatal Zidovudine Prophylaxis. J Acquir Immune Defic Syndr 2007; 44: 106-11. 